\title{
Benefícios da liberação miofascial na cervicalgia
}

\author{
Benefits of myofascial release in cervicalgia
}

Beneficios de la liberación miofascial en cervicalgia

Recebido: 03/11/2021 | Revisado: 10/11/2021 | Aceito: 16/11/2021 | Publicado: 27/11/2021

\author{
Janderson Ramos dos Santos \\ ORCID: https://orcid.org/0000-0003-4921-8775 \\ Centro Universitário do Norte, Brasil \\ E-mail: drjandersonfisio@gmail.com \\ Natália Gonçalves \\ ORCID: https://orcid.org/0000-0002-3084-9668 \\ Centro Universitário do Norte, Brasil \\ E-mail: fisionataliagoncalve@gmail.com
}

\begin{abstract}
Resumo
A cervicalgia é uma doença musculoesquelética que afeta um número considerável de pessoas, causa lesões e afeta suas atividades diárias. A fáscia é uma espécie de tecido conjuntivo, denso e ininterrupto. Envolve e conecta músculos, estruturas nervosas e órgãos internos, sendo um elemento importante comunicação mecânica entre vários sistemas corporais. A liberação miofascial é uma técnica que envolve um alongamento do complexo miofascial de longa duração e baixa carga, com o objetivo de restaurar o comprimento ideal e reduzir a dor para melhorar a função. O principal objetivo da seguinte revisão é mostrar a importância e relevância da técnica de liberação miosfascial na tratativa dos pacientes com cervicalgia. Portanto, o estudo é caracterizado como uma revisão bibliográfica sistemática, para verificar os efeitos e benefícios da liberação miofascial no tratamento da cervicalgia, sendo realizada por meio de pesquisas através das seguintes bases de dados: The Scientific Electronic Library Online (Scielo), Physiotherapy Evidence Database (PEDro), Literatura Latino Americana e do Caribe em Ciências da Saúde (LILACS) e U. S. National Library of Medicine (PubMed). A liberação miofascial é uma das técnicas da terapia manual com baixo custo e fácil aplicabilidade, sendo eficaz no tratamento da cervicalgia, mas, faz-se necessário a importância da obtenção de pesquisas científicas cada vez mais rigorosas nas pesquisas voltadas à prática dos programas fisioterapêutica, a fim de se obter maior eficiência e riqueza de conteúdo.
\end{abstract}

Palavras-chave: Terapia manual; Liberação miofascial; Cervicalgia.

\begin{abstract}
Neck pain is a musculoskeletal disease that affects a considerable number of people, causes injuries and affects their daily activities. Fascia is a kind of connective tissue, dense and uninterrupted. It involves and connects muscles, nervous structures and internal organs, and is an important element in mechanical communication between various bodily systems. Myofascial release is a technique that involves long-lasting, low-load stretching of the myofascial complex, with the aim of restoring optimal length and reducing pain to improve function. The main objective of the following review is to show the importance and relevance of the myosfascial release technique in the management of patients with neck pain. Therefore, the study is characterized as a literature review to verify the effects and benefits of myofascial release in the treatment of cervical pain, being carried out through research through the following databases: The Scientific Electronic Library Online (Scielo), Physiotherapy Evidence Database (PEDro), Latin American and Caribbean Health Sciences Literature (LILACS) and US National Library of Medicine (PubMed). Myofascial release is one of the techniques of manual therapy with low cost and easy applicability, being effective in the treatment of cervical pain, but the importance of obtaining increasingly rigorous scientific research in research aimed at the practice of physical therapy programs is necessary, in order to obtain greater efficiency and richness of content.
\end{abstract}

Keywords: Manual therapy; Myofascial release; Neck pain.

\section{Resumen}

El dolor de cuello es una enfermedad musculoesquelética que afecta a un número considerable de personas, provoca lesiones y afecta sus actividades diarias. La fascia es una especie de tejido conectivo, denso e ininterrumpido. Involucra y conecta músculos, estructuras nerviosas y órganos internos, y es un elemento importante en la comunicación mecánica entre varios sistemas corporales. La liberación miofascial es una técnica que implica un estiramiento prolongado y de baja carga del complejo miofascial, con el objetivo de restaurar la longitud óptima y reducir el dolor para mejorar la función. El objetivo principal de la siguiente revisión es mostrar la importancia y relevancia de la técnica de liberación miosfascial en el manejo de pacientes con dolor de cuello. Por tanto, el estudio 
se caracteriza por ser una revisión de la literatura, para verificar los efectos y beneficios de la liberación miofascial en el tratamiento del dolor cervical, realizándose mediante investigación a través de las siguientes bases de datos: The Scientific Electronic Library Online (Scielo), Physiotherapy Evidence Database (PEDro), Literatura Latino Americana e do Caribe em Ciências da Saúde (LILACS) e U. S. National Library of Medicine (PubMed. La liberación miofascial es una de las técnicas de terapia manual de bajo costo y fácil aplicabilidad, siendo efectiva en el tratamiento del dolor de cuello, pero es necesaria la importancia de obtener una investigación científica cada vez más rigurosa en investigaciones dirigidas a la práctica de programas de fisioterapia, en con el fin de obtener una mayor eficiencia y riqueza de contenidos.

Palabras clave: Terapia manual; Liberación miofascial; Dolor de cuello.

\section{Introdução}

As doenças musculoesqueléticas, definidas como doenças que afetam o sistema motor, incluindo músculos, ossos, articulações, tendões e ligamentos, estão afetando cada vez mais o mundo. Este efeito pode ser medido pelo número de anos de vida com deficiência. (Greco, 2019). Uma das doenças mais comuns nessa situação é a dor cervical, que é o quarto fator de incapacidade mais comum no mundo. (Hoy et al., 2014).

Ao longo dos anos, diversos fatores fizeram com que as pessoas tivessem problemas de saúde relacionados à coluna vertebral. Se não forem tratadas a tempo, muitas vezes evoluem para doenças crônicas, fazendo com que as pessoas restrinjam as atividades diárias e interferem diretamente na qualidade de vida dos indivíduos. Os principais fatores que causam esse problema são: má postura, exercício impróprio, alterações da coluna devido a sobrecarga ou execução incorreta, problemas genéticos. (Santos \& Joia, 2018).

A coluna cervical é uma parte da coluna com grande mobilidade e pouca estabilidade, por isso é mais provável que sofra lesões. Portanto, a disfunção nesta área é uma condição comum que afeta a maioria das pessoas, manifestada como uma série de sinais e sintomas, incluindo dor, amplitude limitada de movimento físico, alterações de sensibilidade e / ou dor à palpação dos músculos do pescoço. (Ferreira Act, et al., 2013)

Dor na cervical e envolvimento dessa área da coluna são comuns. Estima-se que $22 \%$ a $70 \%$ da população mundial sentirá dor no pescoço em algum momento de suas vidas. A prevalência na população geral é de $2 \%$ a $11 \%$, e a de trabalhadores que relatam dificuldade de locomoção por cervicalgia é de $11 \%$ a $14 \%$. No Brasil, a prevalência de dor cervical ao longo da vida é estimada em 20\%, o que está intimamente relacionado a dados socioeconômicos, como renda e escolaridade. (Greco, 2019).

Evidências científicas mostram que, ao longo do tempo, a terapia manual é benéfica para pacientes com cervicalgia. Nos últimos anos, a fisioterapia tem recebido cada vez mais atenção por suas várias técnicas que promovem a normalização do tecido por meio de movimentos do pescoço, para reduzir a compressão dos nervos e trazer relaxamento aos tecidos moles diretamente relacionado à dor. (Martins, 2021)

A fisioterapia é uma ciência aplicada e seus objetos de pesquisa são todas as manifestações e potencialidades do movimento humano, patológico ou seus efeitos psicológicos e orgânicos, cujo objetivo é proteger, manter, desenvolver ou restaurar a integridade de órgãos, sistemas ou funcionalidade, atendendo a diversos tipos de objetivos relacionados à prevenção, cura e restauração da saúde do paciente. Por sua vez, a terapia manual se destaca como um método de tratamento eficaz projetado para tratar dores, lesões, além de desempenhar um papel na prevenção para outras doenças relacionadas. A liberação miofascial é um recurso de tratamento manual na área da fisioterapia, que pode promover o cisalhamento entre a pele e a fáscia muscular. Esta técnica é realizada por uma combinação de três movimentos: movimentos de deslizamento, fricção pressão da tração. A fáscia é entendida como a rede de tensão do tecido conjuntivo, que envolve todas as estruturas de todo o corpo, como músculos, órgãos internos e endotélio. (Santos \& Joia, 2018). 
A própria fáscia é uma espécie de tecido conjuntivo, denso e ininterrupto. Envolve e conecta músculos, estruturas nervosas e órgãos internos, sendo um elemento importante comunicação mecânica entre vários sistemas corporais. A principal função desse tipo de tecido é transmitir tensão, e o sistema de força vem da função de seu revestimento e estrutura de conexão. Devido à sua alta inervação e às características das células responsáveis por prover estresse e alongamento, esse tecido costuma estar associado a dor. Além disso, auxilia na percepção da postura (Oliveira, et al, 2019).

Um conjunto de fáscia que gera componentes biológicos de tensão, neste processo a transmissão de força estabiliza a estrutura e distribui uniformemente a carga de todo o corpo. Devido a esta característica, o tecido auxiliar da fáscia é estabilizado, por envolver estrutura, é responsável pela estabilização dinâmica do sistema musculoesquelético. (Moccia, et al, 2015).

É chamada de membrana de tecido conjuntivo e fica logo abaixo a pele permite que os músculos deslizem suavemente durante os exercícios básicos e esportes. A fáscia tem a função de ajudar a manter a força muscular, pois sustenta os músculos, permitindo que se contraiam de forma mais eficaz, permitindo que os músculos deslizem entre si com facilidade, de modo que a tensão gerada pela atividade muscular reduz o atrito quando envolvido cada músculo como uma membrana de proteção. (Sullivan, et al, 2013)

A fáscia, juntamente com o músculo e o tecido conjuntivo, constituem a maior parte do tecido. Afetadas por lesões na prática de atividades esportivas, essas lesões estão relacionadas a micro traumas contínuos ou grandes traumas teciduais, levando a processos inflamatórios e tornando está desordem tecidual reticular e formar aderências e fibrose. (MARTINS, et al, 2019).

Cada subcamada de fibras de colágeno é paralela A própria direção e a direção entre as fibras das camadas adjacentes mudam, formando um ângulo de cerca de 70-80 graus entre si. Isso permite que folhas de fáscia mais densas deslizem livremente nas camadas subjacentes sem atrito significativo e aumenta a capacidade da fáscia de suportar tensões em quase todas as direções. (Benetazzo, et al, 2011).

Problemas neuromusculares são causados por mudanças na fáscia, por sua vez, alteram os fusos musculares que estão diretamente ligados ao sistema nervoso, portanto, a fáscia muda de acordo com os sistemas nervoso e emocional, pois como sabemos, os comandos do nosso corpo vêm desses sistemas. Outros problemas relacionados, como acidentes, traumas, infecções, etc., também podem causar alterações na fáscia, o que pode causar dor dependendo da situação, pode variar de aguda a crônica. (Sousa, et al., 2017).

A região da coluna cervical está localizada na parte inferior do crânio, que é a base de suporte onde se localiza o pescoço, sendo composta por ossos, músculos e artérias com funções vitais. A coluna cervical consiste nas primeiras sete vértebras, localizadas diretamente abaixo do crânio e é mais flexível do que as outras partes da coluna (coluna torácica e lombar). (Santos \& Joia, 2018).

É composto por sete vértebras, as duas primeiras (atlas e áxis) apresentam características anatômicas que são diferentes das outras. O atlas é em forma de anel, não tem corpo vertebral, está conectado à base do crânio através da articulação atlanto-occipital, responsável pela maior parte do movimento sagital da coluna vertebral. $\mathrm{O}$ áxis, a segunda vértebra, tem uma parte saliente que se projeta de seu corpo chamado de processo odontóide, projetando-se para o atlas formam um pivô, onde a articulação atlanto-axial realiza as rotações do crânio, não há disco entre essas duas vértebras, separados e apoiados por vários ligamentos internos. (Natour, 2004)

Atlas, é a primeira vértebra cervical é responsável por sustentar a cabeça, é articulada com o áxis, permitindo assim os amplos movimentos, outra característica marcante é o fato de não possuir corpo vertebral. Sua posição anatômica: fóvea dental é anterior; face articular superior (a maior) é superior. Áxis, a segunda vértebra cervical está em contato direto com o atlas formando assim um eixo de rotação para a cabeça. Possui uma característica que o distingue facilmente das demais vértebras, o 
seu dente, graças a esse tipo de articulação podemos fazer o movimento de rotação da cabeça. Sua posição anatômica: o dente é anterior e superior. Sétima vértebra cervical, é bem parecida com as demais, porém por possuir um processo espinhoso longo e proeminente, sendo esta sua característica especial. Sua posição anatômica: o processo espinhoso é posterior e inferior. (Portela, 2016, p. 36).

Mobilidade é a capacidade de se mover livremente; uma boa mobilidade articular requer uma contração e relaxamento eficazes dos músculos ao redor das articulações para alcançar um movimento suave. A mobilidade da coluna varia de região, na região da coluna cervical e lombar tem maior mobilidade, enquanto a da coluna torácica não é muito flexível. (Willardson, 2017).

O grupo pré-vertebral são músculos profundos que consiste no músculo longo do pescoço, longo da cabeça, reto anterior da cabeça e reto lateral do pescoço. Na contração unilateral desses músculos acontece a flexão lateral do pescoço e rotação da cabeça. No entanto, na contração bilateral eles executam a flexão do pescoço. Esternocleidomastoide, um músculo superficial constituído de duas cabeças, atua unilateralmente realizando a flexão lateral do pescoço. Os músculos escalenos, embora considerado anterior, situam-se mais lateralmente, tendo sua importância na respiração. O músculo esplênio, parte dos eretores da espinha, é atuante na extensão das vértebras cervicais, um músculo achatado situado na parte superior do dorso e posterior do pescoço. O semiespinhal consiste em finos fascículos que possuem tendões longos em suas extremidades, originam-se dos dois processos espinhosos cervicais e dos quatro processos espinhosos torácicos. (Portela, 2016, p. 42)

A principal função da coluna cervical é apoiar e guiar a cabeça no espaço relativo ao tórax que serve ao sistema sensorial. Esta tarefa requer um sistema musculoesquelético complexo para combinar mobilidade e estabilidade, tendo ligação a fatores externos e internos facilitando a disfunção da área cervical. (Silva, 2017).

Estudos têm mostrado que a relação entre esforço, repetição e exercício de sobrecarga estática pode levar a várias doenças miofasciais e esqueléticas. Essas doenças podem estar relacionadas à contração contínua, redução do suprimento sanguíneo e compressão dos feixes nervosos, o que pode causar dor. Então, quando as partes do corpo não estão bem conectadas, não existe equilíbrio entre eles, o faz responder mal a certos estímulos e ações, levando a uma variedade de disfunções. (Santos \& Joia, 2018).

Esse estudo se faz necessário devido as incidências de casos de cervicalgia na população global que sofre dessa patologia. Por tanto, busca-se justificar os benefícios proporcionados através da terapia manual pela técnica de liberação miofascial, para promover a melhora da qualidade de vida, redução do quadro álgico e melhorar o desempenho das atividades de vida diária.

\section{Metodologia}

O estudo é caracterizado como uma revisão bibliográfica sistemática sendo realizada por meio de pesquisas através das seguintes bases de dados: The Scientific Electronic Library Online (Scielo), Physiotherapy Evidence Database (PEDro), Literatura Latino Americana e do Caribe em Ciências da Saúde (LILACS) e U. S. National Library of Medicine (PubMed). Foram analisados os trabalhos referente ao tema dos benefícios da liberação miofascial na cervicalgia, sendo entre os anos 2011 até 2021. Com os seguintes critérios de delimitações: fisioterapia, terapia manual, liberação miofascial, cervical, cervicalgia em português. Physiotherapy, manual therapy, myofascial release, cervical, cervical pain em inglês.

Os critérios utilizados para inclusão foram: tratamento fisioterapêutico para cervicalgia, benefício da liberação miosfascial, prática fisioterapêutica com uso de terapia manual, importância da liberação miofascial no tratamento da cervicalgia. Assim, foram considerados todos os artigos que se relacionavam com o perfil do paciente avaliado. Sendo selecionados 5 artigos que relacionavam com o conteúdo avaliado. 
O perfil de exclusão foram trabalhos fora de contexto e anos anteriores pré-estabelecidos. Artigos que direcionavam a técnica de liberação miofascial para outros segmentos corporais que sofrem disfunções musculoesqueléticas, como: tórax, lombar e quadril.

\section{Resultados e Discussão}

A dor na coluna pode estar relacionada a diferentes patologias, portanto, a dor na cervical é considerada um sintoma e deve ser investigada para se obter um diagnóstico preciso para que possa ser tratada. (Marcleice et al., 2012)

A dor na cervical é muito comum e o diagnóstico é complexo, pois pode estar relacionada a uma variedade de causas. Podem ser devido a condições profissionais, disfunção muscular, espondilose, síndrome do desfiladeiro torácico, tumores da coluna vertebral, artrite reumatoide, infecções e fraturas. No entanto, na maioria dos casos, a causa não é específica. (Borges et al., 2013)

A dor no pescoço é caracterizada ao nível da coluna cervical, a área entre a base do osso occipital e a torácica cervical. Pode ser agudo ou crônico está relacionado a doenças biomecânicas e musculares, causando dor, inflamação e dor em queimação. Pode haver restrições ao movimento passivo do pescoço e alterações no tecido muscular do pescoço. (Pereira et al., 2016)

Afeta um número considerável de indivíduos, em média $12 \%$ a $34 \%$ dos adultos encontram-se em determinada fase da vida, as mulheres apresentam maior incidência e afetam as atividades da vida diária. (Regina et al., 2017). Um fato importante a se considerar é a incidência nas mulheres têm mais dores no pescoço do que os homens, o que pode estar relacionado ao acúmulo de mais tarefas diárias, e a maioria também sai para trabalhar além das atividades familiares, levando ao excesso de atividade. No Brasil, as mulheres têm maior probabilidade de serem diagnosticadas com problemas crônicos de coluna (21,1\%) do que os homens (15,5\%). (IBGE, 2013)

A região do pescoço apresenta grande mobilidade, o que leva à fadiga e degeneração da estrutura da região ricamente inervada. O diagnóstico é baseado na apresentação clínica apresentada pelo paciente. Os exames de imagem (como raios X, ressonância magnética, tomografia computadorizada) ajudam a determinar a extensão da lesão e sua localização exata. (Meziat; Silva, 2011)

A dor na cervical raramente começa rapidamente, geralmente está relacionada a movimentos repentinos, postura inadequadas de longo prazo, esforço ou trauma. Pode ser definida como dor localizada na nuca e na parte superior da escápula ou na parte superior das costas. Portanto, na maioria dos casos, a dor no pescoço é o resultado de movimentos repetitivos, má postura ou esforço excessivo. (Pereira, et al, 2016).

A principal manifestação clínica da dor cervical é a dor do tipo choque, que segue ao longo do caminho da raiz e piora com o movimento da raiz distendida, tórax ou coluna vertebral. As parestesias podem ocorrer na extremidade distal da raiz. Mudanças nos reflexos, tônus, força ou mudanças nutricionais podem estar ausentes ou atrasadas. As manifestações clínicas incluem dor no pescoço, mobilidade restrita e diminuição da força dos membros superiores. (Marcleice et al, 2012).

A terapia de liberação miofascial é uma terapia manual que envolve um alongamento do complexo miofascial de longa duração e baixa carga, com o objetivo de restaurar o comprimento ideal e reduzir a dor para melhorar a função. Acreditase que, por meio do alongamento restrito da fáscia, a liberação miofascial pode normalizar as propriedades de comprimento e deslizamento do tecido miofascial, ao mesmo tempo que libera a pressão da sensibilidade da estrutura, restaurando assim a mobilidade muscular. (Greco, 2019).

Diversas alternativas de tratamento têm sido propostas para o manejo da disfunção facial. Nos últimos anos, o crescente interesse no sistema miofascial levou a um aumento nas pesquisas sobre a eficácia da terapia de liberação miofascial na redução da dor e na correção da postura. (Picelli, et al, 2011). 
A liberação miofascial é realizada por uma combinação de movimentos de tração, como deslizamento, fricção e amassamento. O objetivo é alongar os músculos e a fáscia e relaxar os tecidos tensos. A técnica envolve a aplicação de sobrecarga aos tecidos moles, estimulando os mecanorreceptores na fáscia, então a barreira que produz resistência é atacada e o tecido estica, o que é considerado um movimento involuntário porque é realizado manualmente por um fisioterapeuta. (Santos \& Joia, 2018).

Esses movimentos podem ser definidos como internos ou externos e, quando se caracterizam como internos, são considerados movimentos inertes, ritmos corporais, como contração muscular, movimentos dos olhos, etc. Quando são externos, o terapeuta aplica força, compressão e torção para induzir uma leve tensão nos tecidos moles, o que pode causar algumas reações positivas do paciente. (Silva, 2017). Muitos benefícios podem ser mencionados, por exemplo, auxilia na atividade física, aumenta a mobilidade articular, auxilia na prática de exercícios, alivia a tensão, ativa os músculos e promove a recuperação muscular, previne lesões, entre outros benefícios. (Marcleice et al, 2012).

Um estudo mostrou que a MLT reduziu em $20 \%$ a dor e os limiares de dor à pressão dos músculos trapézios e do baço em pacientes com dor no pescoço em comparação com um plano de fisioterapia multimodal. Aproximadamente 23,33\% dos pacientes com dor cervical apresentam alterações "fundamentais" no deslizamento da fáscia. (Tozzi, 2011).

O principal objetivo da técnica liberação miofascial é reduzir a adesão da fibra na rede da fáscia e restaurar a função dessas redes. Os efeitos diretos no corpo incluem o alívio da dor, melhora do desempenho atlético, aumento da flexibilidade e promoção do movimento, bem como mudanças mais subjetivas, como melhora da postura. (Shah; Bhalara, 2012).

A fisioterapia pode desempenhar um papel importante no tratamento de pacientes com dor cervical, pois visa reduzir a dor, restaurar a mobilidade e fortalecer os músculos, melhorando assim a qualidade de vida. A liberação miofascial é uma técnica de terapia manual, uma especialidade da fisioterapia que ajuda a tratar a dor no pescoço. Portanto, entende-se que a intervenção terapêutica manual é benéfica para o tratamento da dor cervical.

Florentino et al. (2012), concluíram que a liberação miofascial é eficaz no tratamento da cervicalgia, mas de fato sua eficácia já foi comprovada quando relacionada a outros tipos de tratamento com ou sem medicamentos. Portanto, as intervenções de fisioterapêutica incluindo exercícios de alongamento, técnicas de relaxamento e massagem são benéficas para melhorar a qualidade de vida e flexibilidade para pacientes com dor na cervical.

Borges et al. (2013), observaram que 4 semanas de exercícios de alongamento relacionados à terapia manual podem reduzir a dor cervical e a limitação articular em 52\%. A importância da técnica de alongamento neste tipo de tratamento é eficaz e melhora a eficiência trazida pela combinação dos dois métodos, enfatizando novamente a combinação de LM e outros tipos de tratamentos produzindo melhores resultados.

Borges et al. (2013), em relação ao impacto da fisioterapia na qualidade de vida dos pacientes com cervicalgia, podem ser analisados os seguintes resultados: melhoria da qualidade de vida e abrangência exercício adulto, aspectos físicos e psicológicos, nível de independência, relações sociais e aspectos ambientais da qualidade de vida e amplitude de movimento. Foram significativamente melhorados após a fisioterapia em flexão e extensão da coluna cervical, lateralização e rotação. Portanto, conclui que as intervenções fisioterapêuticas incluindo exercícios de alongamento, técnicas de relaxamento, massagem e eletroterapia são eficazes, sendo benéficos a melhorar a qualidade de vida e a flexibilidade de pacientes com dor cervical.

Sousa (2015) diz que, por meio da técnica de liberação miofascial, dentre as variáveis analisadas, ao reduzir a intensidade subjetiva geral, efeitos significativos foram alcançados no tratamento das cefaleias tensionais alívio da dor, sensação e componentes emocionais. Relatam ainda que, mesmo não havendo um plano fisioterapêutico exato para o tratamento das cefaleias tensionais, a técnica LM utilizada têm se mostrado satisfatórias e resolvidas.

Uemura et al. (2019), evidenciaram que a liberação miofascial, seja manual por instrumentos ou auto liberação, pode 
trazer benefícios aos praticantes de atividades esportivas, tais como: aumento da flexibilidade, amplitude de movimento, força e do limiar de dor.

\section{Considerações Finais}

Considerando a classificação e a etiologia da patologia, o estudo propôs uma definição de cervicalgia, descrevendo sobre a anatomia da coluna cervical, incidência e fisiopatologia. A liberação miofascial foi escolhida como método de tratamento porque objetivo resumir o tratamento de reabilitação de pacientes com dor cervical. Ao analisar os resultados obtidos em artigos sobre o tratamento da cervicalgia por meio da técnica de liberação miofascial, conclui-se que a liberação miofascial é eficaz no tratamento da cervicalgia.

A pesquisa neste artigo nos levou a concluir que a liberação miofascial é uma estratégia eficaz para pacientes com dor na cervical para ganhar flexibilidade, melhorar os exercícios, aliviar a dor e aprimorar a capacidade funcional. Ressaltando a importância da obtenção de mais novas pesquisas científicas, voltadas aos recursos fisioterapêuticos sobre a técnica de liberação miofascial, a fim de se obter maior eficiência e riqueza de conteúdo.

Para desfechos de sugestões, é importante destacar a quantidade de conteúdo sobre a técnica de liberação miofascial, sendo um recurso da terapia manual no auxílio das disfunções musculoesqueléticas, sendo de suma importância para os profissionais da área de fisioterapia que utilizam dessa técnica em suas condutas terapêuticas.

\section{Agradecimentos}

O desenvolvimento deste trabalho de conclusão de curso contou com a ajuda de diversas pessoas, dentre as quais eu agradeço: Primeiramente a Deus pelas bênçãos e conquistas de todos os dias. Aos meus pais (Lúcio Sousa e Maria de Jesus) e irmão (Jarlyson Ramos) que me ensinaram a nunca desistir, sempre me incentivando a buscar o meu melhor e prezaram a minha educação, minha noiva (Jéssica Santana) por também ser uma das incentivadoras estando ao meu lado, meu supervisor (Manoel Eufrásio) e colegas de trabalho por me ajudarem e apoiar quando precisei, a todos os professores que puderam repassar os seus conhecimentos para me tornar um profissional ético e moral. Ao Dr. Alessandro de Souza fundador da clínica Doctor Fisio, onde pude estagiar e ter a experiência e ensinamentos, deixo meus sinceros pêsames a família pelo falecimento do Dr sendo mais uma vítima da covid-19. E aos meus colegas de turma que caminhamos juntos até o fim dessa graduação.

\section{Referências}

Benetazzo, L., Bizzego, U., Caro, R., Frigo, G., Guilolin, D. \& Stecco, C. (2011). 3D reconstruction of the crural and thoracolumbar fasciae. Anatomia Cirúrgica e Radiológica, 33(10):855-62. DOI: 10.1007/s00276-010-0757-7. https://pubmed.ncbi.nlm.nih.gov

Borges, M. C., Borges, C. S., Silva, A. G. J., Castellano, L. R.C. \& Cardoso, F.A.G. (2013). Evaluation of quality of life and the physiotherapy treatment in patients with chronic neck pain. DOI: 10.1590/s0103-51502013000400016. https://www.scielo.org

Greco, R. C. (2019). Efeitos da mobilização de tecidos moles assistida por instrumentos (IASTM) na dor e incapacidade em indivíduos com cervicalgia crônica inespecífica. Um ensaio clínico randomizado. https://repositorio.ufcspa.edu.br

Silva, D. A. M., Soares, F. B. M., Oliveira, P. M., Silva, A. C. M., Sales, A. O., Porto, R. E. A. \& Galera, S. R. G. P. (2017). Tratamento da cervicalgia mecânica por meio das técnicas de tração e pompage: relato de caso. Revista Ciência Saúde 2017;2(3):8-12. https://revistaeletronicafunvic.org

Sousa, R. C. (2015). Efeitos da liberação miofascial na qualidade e frequência da dor em mulheres com cefaleia do tipo tensional induzida por pontos-gatilho. Fisioterapia Brasil, v. 16, n. 3, p. 231-235. DOI: https://doi.org/10.33233/fb.v16i3.80

Santos, H. A. \& Joia, L. C. (2018). A liberação miofascial nos tratamentos de cervicalgia. Revista das Ciências da Saúde do Oeste Baiano - Higia, 2018; 3 (1): 151-167. https://fasb.edu.br

Ferreira, A. C. T., Martini, F. A. N. \& Pires, P. F. (2013). Comparação da Amplitude do Movimento Cervical em Mulheres com Disfunção Cervical e Assintomáticas. Saúde em Revista, v. 13, n. 33, p. 31 - 37. DOI: http://dx.doi.org/10.15600/2238-1244/sr.

Florentino, D. M., Sousa, F. R. A., Maiworn, A. I., Carvalho, A.C.A. \& Silva, K.M. (2012). A fisioterapia no alívio da dor: uma visão reabilitad ora em cuidados paliativos. Revista do Hospital Universitário Pedro Ernesto, UERJ. https://e-publicacoes.uerj.br 
Research, Society and Development, v. 10, n. 15, e334101522724, 2021

(CC BY 4.0) | ISSN 2525-3409 | DOI: http://dx.doi.org/10.33448/rsd-v10i15.22724

Hoy, D., March, L., Woolf, A., Blyth, F., brooks, P., Smith, E., Vos, T., Barendregt, J., Blore, J., Murray, C., Burstein, R. \& Buchbinder, R. (2014). The global burden of neck pain: estimates from the Global Burden of Disease 2010 study. Annals of the Rheumatic Diseases, v. 73, n. 7, p. 1309-1315. DOI: 10.1136/annrheumdis-2013-204431

IBGE. (2013). IBGE | Biblioteca | Detalhes | Pesquisa nacional de saúde: 2013, percepção do estado de saúde, estilos de vida e doenças crônicas: Brasil, grandes regiões e unidades da federação. p. 155 -164. https://biblioteca.ibge.gov.br

Marcleice, R. V. \& Mejia, D. P.M. (2012). As terapias manuais e a cinesioterapia no tratamento da cervicalgia. https://portalbiocursos.com.br/

Martins, A. P., Pereira, K. P. \& Felicio, L R. (2019). Evidências da técnica de liberação miofascial no tratamento fisioterapêutico: revisão sistemática. Arquivos de Ciência do Esporte. DOI: https://doi.org/10.17648/aces.v7n1.3504

Martins, S. M. P., Melo, A. C. \& Alencar, I. (2021). The benefits of manual therapies in tension headache: a literature review. Research, Society and Development, v.10, n.12, e470101220824, 2021. https://rsdjournal.org/

Meziat, N. F. \& Silva, G. A. (2011). Invalidez por dor nas costas entre segurados da Previdência Social do Brasil. Revista de Saúde Pública, v. 45, n. 3, p. 494-502, jun. 2011. DOI: 10.1590/S0034-89102011000300007. https://www.scielo.org

Moccia, D., Nackashi, A. A., Schilling, R. \& Ward, P. J. (2015). Fascial bundles of the infraspinatus fascia: anatomy, function, and clinical considerations. DOI: 10.1111/joa.12386. https://pubmed.ncbi.nlm.nih.gov

Natour, J. (2004). Coluna vertebral: Conhecimentos básicos. Etecetera Editora de Livros e Revistas. 2004, p. 21-43.

Oliveira, A. P. M., pereira, K. P. \& Felicio, L. R. (2019). Evidências da técnica de liberação miofascial no tratamento fisioterapêutico: revisão sistemática. Arquivos de Ciências do Esporte, v. 7, n. 1, 2019.DOI: https://doi.org/10.17648/aces.v7n1.3504

Pereira, C. S., Pedra, F. L. S., Silva, F. H., Castro, N. A., Silva, V. F. \& Venturini, C. (2016). Medo de cair em idosos com dor cervical comparado aos idosos assintomáticos. Revista Sinapse Múltipla, v. 5, n. 2, p. 90-90, 2016.

Pereira, J., Cesca, D., Daronco, L. S. E. \& Balsan, L. A. G. (2016). Efeitos do tratamento quiroprático na concentração sérica de proteína creativa e nos sintomas de indivíduos com cervicalgia. Salusvita, Bauru, v. 35, n. 2, p. 243-257, 2016. https://secure.unisagrado.edu.br

Picelli, A., Ledro, G., Turrina, A., Santilli, V. \& Smania, N. (2011). Effects of myofascial technique in patients with subacute whiplash associated disorders: a pilot study. Eur J Phys Rehabil Med. 2011 Dec;47(4):561-8. Epub 2011 Jul 28. PMID: 21796089. https://pubmed.ncbi.nlm.nih.gov

Portela, J. P. (2016). Cinesiologia. Sobral, $1^{\circ}$ edição, 2016, p. 33-46. https://md.uninta.edu.br

Shah, S. \& Bhalara, A. (2012). Myofascial Release. International Journal of Health Sciences \& Research, v. 2, n. 2, p. 69, 2012. https://www.ijhsr.org

Sousa, L., Araújo, V. A., Sauza, E. S., Santos, R. M. C., Mendonça, W. V., Arruda, J. L. R. \& Cruz, R. A. R. S. (2017). Auto liberação miofascial x Alongamento estático: Efeitos sobre a flexibilidade de escolares. Revista CPAQV - Centro de Pesquisas Avançadas em Qualidade de Vida - CPAQV Journal, v. 9 , n. 2, 2017. https://www.cpaqv.org

Sullivan, K. M., Silvey, D. B. J., Button, D. C. \& Behm, D. G. (2013). Roller-massager application to the hamstrings increases sit-andreach range of motion within five to ten seconds without performance impairments. Int $J$ Sports Phys Ther. Jun, 2013 p. 228-36. PMCID: PMC3679629. https://pubmed.ncbi.nlm.nih.gov

Tozzi, P., Bongiorno, D. \& Vitturini, C. (2011). Fascial release effects on patients with non-specific cervical or lumbar pain. Journal of Bodywork and Movement Therapies. DOI: 10.1016/j.jbmt.2010.11.003 https://pubmed.ncbi.nlm.nih.gov

Uemura, G. T., Prieto, F. F. S. \& Saciloto, M. R. R. (2019). Os principais benefícios da aplicação de liberação miofascial em praticantes de atividades físicas. Rev. InterCiência-IMES Catanduva, v. 1, n. 2, p. 27-27, 2019. https://www.fafica.br

Willardson, J. M. (2017). Desenvolvendo o core. Editora Phorte; ISBN: 8576556197; São Paulo, 2017, p. 16 - 20. https://issuu.com 